\title{
Automated Analysis of Eye Tracking Movements
}

\author{
Adrian Ruetsche $^{a} \quad$ Ann Baumann ${ }^{a}$ Xiaoyi Jiang ${ }^{a, c}$ Daniel S. Mojon a,b \\ a Laboratory for Experimental Oculography and 'bepartment of Strabismus and Neuro-Ophthalmology, \\ Kantonsspital, St. Gallen, Switzerland; 'Department of Electrical Engineering and Computer Science, \\ Technical University of Berlin, Berlin, Germany
}

\section{Key Words}

Photo-oculography · Visual pursuit - Eye movement recording - Data analysis - Diagnostic techniques • Statistics

\begin{abstract}
Objective: To present a quick algorithm to automatically analyze the raw data acquired by a photo-oculography (POG) system. Methods: We developed a simple algorithm for POG data analysis based on an extrapolation of missing values due to blinking and on exclusion of outliers using the robust mean and standard deviation. Results: POG curves of 4 children aged between 1.5 and 7 years are shown before and after automatic analysis. After applying our algorithm, the curves are much smoother. Conclusion: Our algorithm allows a quick data analysis and will help to better interpret and analyze POG data.
\end{abstract}

Copyright ( $\odot 2003$ S. Karger AG, Basel

\section{Introduction}

Oculomotor responses such as fixation behavior, optokinetic nystagmus and visual pursuit represent good modalities to study visual functions, even in nonverbalized infants [1]. Since observation of the responses alone does not allow an objective assessment, several techniques for quantitative eye movement recording have been proposed. Photo-oculography (POG) is based on the measurement of the relative position of the reflected image of an infrared source on the cornea and the pupil center. Electro-oculography measurements are performed with cutaneous electrodes using the dipole propriety of the eye. In the scleral search coil technique, the position of a contact lens coil is determined. POG is widely used, since the method is more sensitive and precise than electro-oculography and since it lacks the discomfort and risk of corneal abrasion with the scleral search coil technique. Disadvantages of POG measurements include blinking artifacts, pupil masquerade by the eyelid and loss of the reflected image secondary to larger head movements [2]. Although different POG systems exist, to our knowledge, all methods of data analysis are proprietary. However, without being familiar with the exact algorithms used for data analysis, interpretation and further statistical processing of the results are difficult. In this study, we propose for smooth pursuit eye movements an algorithm to automatically analyze the raw data acquired by a POG system.

\begin{tabular}{ll}
\hline KARGER & ( ) 2003 S. Karger AG, Basel \\
0030-3755/03/2175-0320\$19.50/0 \\
$\begin{array}{l}\text { Fax +4161306 1234 } \\
\begin{array}{l}\text { E-Mail karger@karger.ch } \\
\text { www.karger.com }\end{array}\end{array}$ & $\begin{array}{l}\text { Accessible online at: } \\
\text { www.karger.com/oph }\end{array}$
\end{tabular}

Daniel Mojon, MD

Department of Strabismus and Neuro-Ophthalmology

Kantonsspital

CH-9007 St. Gallen (Switzerland)

Tel. +41 7149428 20, Fax +41 7149428 82, E-Mail daniel.mojon@kssg.ch 


\section{Methods}

This research followed the tenets of the Declaration of Helsinki and was approved by the Ethical Committee of Kantonsspital St. Gallen. An extensive description of the experimental setting has been published previously [1-3]. The stimulus generator and registration system is provided by Metrovision ${ }^{\circledR}$ (Perenchies, France).

\section{Subjects}

Smooth pursuit eye movements were measured in 4 children (aged 1 year 6 months, 3 years 3 months, 3 years 5 months and 6 years 9 months). All children were healthy and full-term. An orthoptic examination included the Hirschberg ocular alignment test, cover test, four-prism diopter base out fusion test and pupillary reaction, visual acuity measurements in each eye, and stereoscopic tests including the Lang stereotest were normal. Infants were examined in Prechtl's [4] state III (calm wakefulness with open eyes, regular breathing, absence of gross body movements), because more reliable results are obtained from infants in this behavioral state [3].

\section{Materials and Methods}

Stimulus Presentation. Infants and toddlers up to the age of 1.5 years were seated in an infant car seat $\left(\right.$ Maxicosy $\left.{ }^{\circledR}\right)$ with an inclination of $45^{\circ}$ (fig. 1). This inclination minimizes pupil masquerade by the eyelid [2]. Their heads were not stabilized. Children between 1.5 and 6 years were seated on the lap of the mother or alone, with their heads stabilized by a chin and front rest (fig. 2). In both settings, the monitor for stimulus presentation was placed in a frontoparallel position at a distance of $40 \mathrm{~cm}$ from the eyes.

Eye Movement Recordings. Eye position was determined by measuring the position of the corneal reflex with respect to the center of the pupil. Eye movements were registered under binocular viewing conditions from the right eye. An infrared illumination of the eye $(880 \mathrm{~nm})$ was used to produce the corneal reflex and the pupil image. The system operated with a sampling rate of $30 \mathrm{~Hz}$ and achieved a resolution of 10 arc minutes [5]. Illumination source and camera were installed above the children's head. A hot mirror (dichroic filter separating visible light and infrared light) was used to illuminate the eye and to register the reflexes with a camera. Calibration was defined by the geometry of the anterior chamber [1]. It was estimated from biometry data of eyes of subjects obtained at the same age as the subjects used in our study. Optimal alignment during registration was achieved looking at an image of the child's eye on a computer screen. If necessary, the head position of the child was adjusted during the registration period.

Stimulus Characteristics. Colored (red, violet, blue, yellow, white, green) $1.2^{\circ} \times 1.2^{\circ}$ squares were moving horizontally or vertically at a constant velocity of either $7.5,15$ or $30^{\circ}$ s against a uniform gray background. Different colors were used in order to achieve a better attentiveness of the children. At the screen's edges a pause of $333 \mathrm{~ms}$ (square does not move) prevented the subjective impression that the stimulus moves faster when changing its direction. The corresponding amplitudes were $26.6^{\circ}$ horizontally and $20.6^{\circ}$ vertically. One test cycle (registration time) lasted $38 \mathrm{~s}$. Using the stimulus characteristics it was possible to build a two-dimensional stimulus curve for each combination of stimulus velocity and direction which was essential for data analysis. The following coordinate system was used: $\mathrm{x}$-coordinate $=$ horizontal, $\mathrm{y}$-coordinate $=$ vertical, negative values $=$ to the left or inferior to the center, positive values $=$ to the right or superior to the center.

Automated Analysis of Eye Tracking

Movements

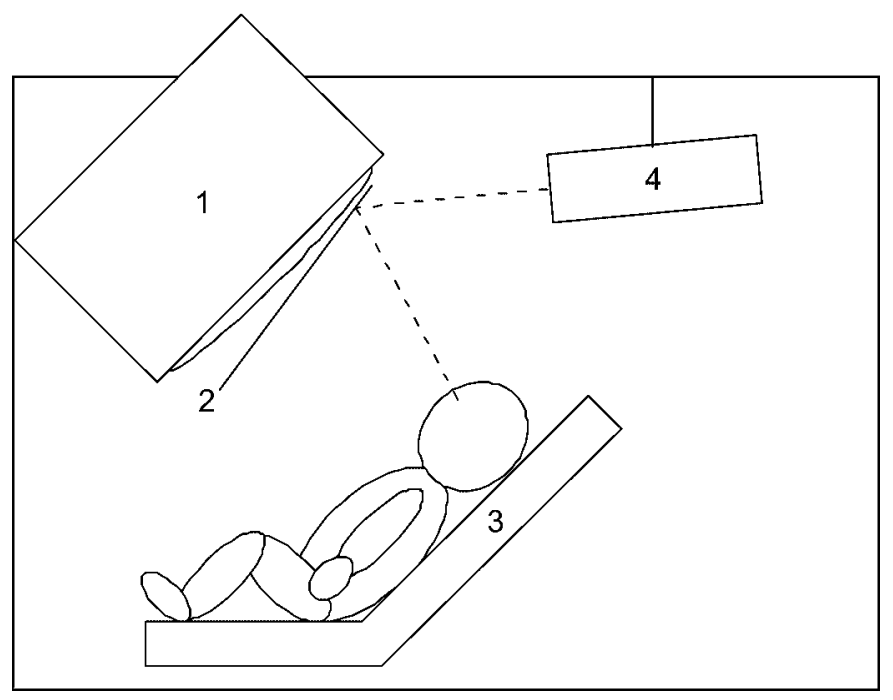

Fig. 1. Examination technique for infants and toddlers up to the age of 1.5 years: $1=$ monitor for stimulus presentation, $2=$ hot mirror, $3=$ infant car seat, $4=$ infrared illumination and camera.

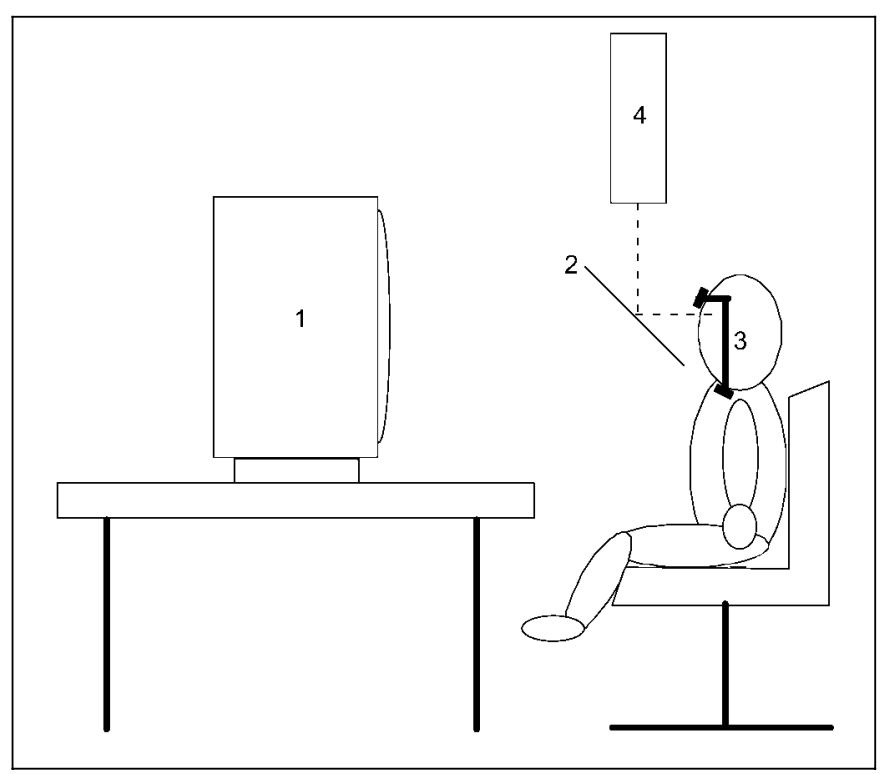

Fig. 2. Examination technique for children older than 1.5 years: $1=$ monitor for stimulus presentation, $2=$ hot mirror, $3=$ chin and front rest, $4=$ infrared illumination and camera.

Calibration. Correction factors were derived from repeated presentations to a large number of children of a vertical and horizontal step ramp stimulus with the following $\mathrm{x} / \mathrm{y}$-axis: horizontal step ramp $0^{\circ} /-10^{\circ}, 0^{\circ} / 0^{\circ}, 0^{\circ} \%+10^{\circ}$ and vertical step ramp $-10^{\circ} / 0^{\circ}, 0^{\circ} / 0^{\circ},+10^{\circ} / 0^{\circ}$ [1].

Registered Data. Registration supplies the running time in seconds, the $\mathrm{x}$ - and y-coordinates of the eye's position in degrees with a frequency of $30 \mathrm{~Hz}$. The duration of smooth pursuit registration of 

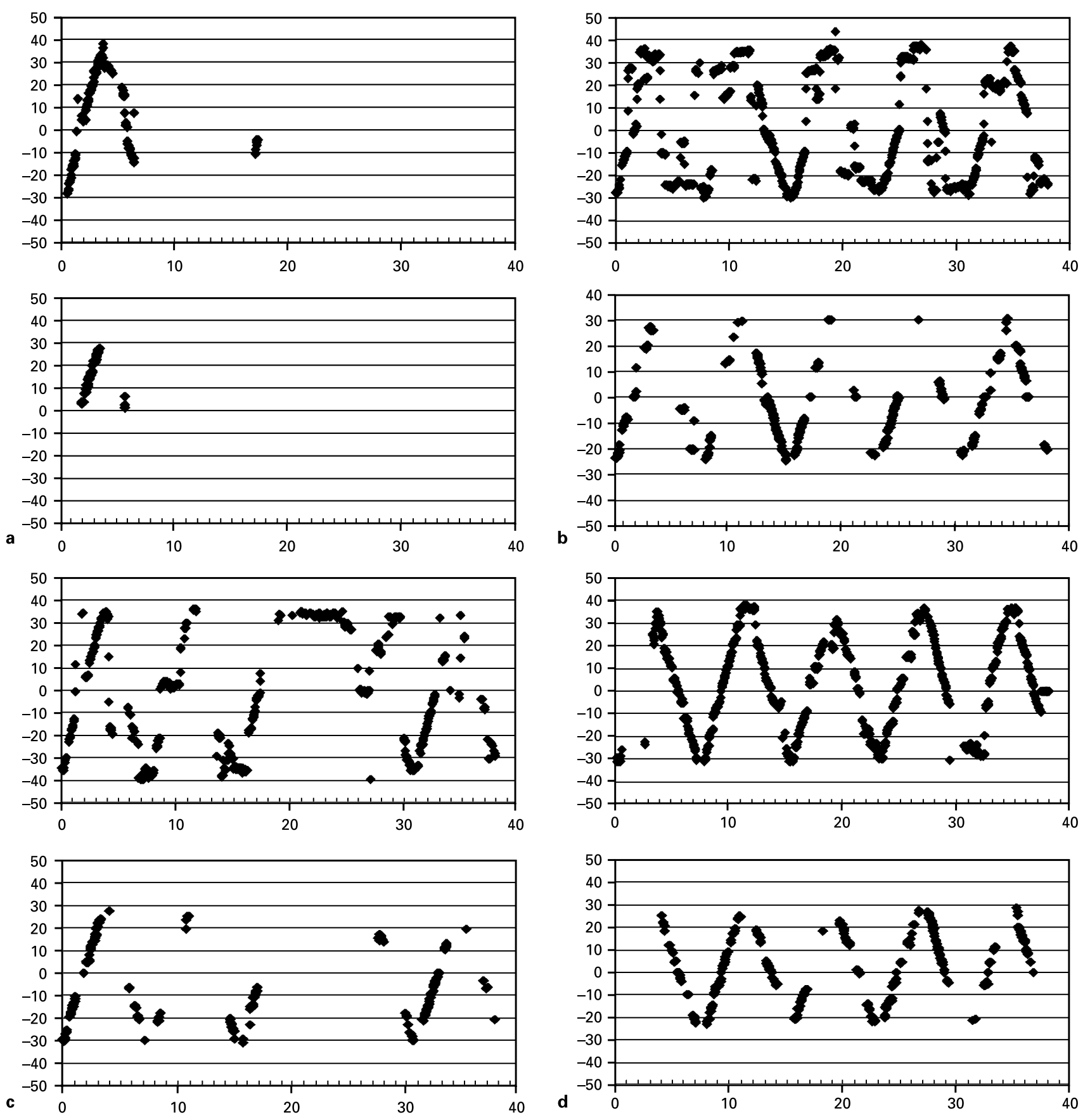

Fig. 3. Curves of horizontal pursuit movements (stimulus velocity $15 \%$ ) of 4 children before (upper curves) and after (lower curves) applying our algorithm. The abscissa corresponds to time (seconds), the ordinate to horizontal eye position (degrees), with negative values $=$ to the left of the center, positive values $=$ to the right of the

center. a Child aged 1 year and 6 months with poor cooperation. Only a registration of about $7 \mathrm{~s}$ was possible. $\mathbf{b}$ Child aged 3 years and 3 months with good cooperation. c Child aged 3 years and 6 months with rather good cooperation. d Child aged 6 years and 9 months with very good cooperation. 
$38 \mathrm{~s}$ resulted in 1,140 lines in the data file. During the pauses of the stimulus at the screen's edge, data are excluded from analysis, as they do not contribute to smooth pursuit eye movements. Because of the variability of the oculomotor response to the change of the stimulus direction, additionally, all data deriving from a margin of $2.32^{\circ}$ from the screen's edge are excluded.

\section{Analysis of Eye Tracking Movement Data}

\section{Extrapolation of Missing Point}

Due to blinking or loss of interest a variable amount of points may be missing. The average loss of data points by blinking was determined in normals and found to be 3 measurements $(0.1 \mathrm{~s})$, as was also suggested in other studies [6]. Therefore, gaps of up to 3 data points are extrapolated for the $\mathrm{x}$ - and $\mathrm{y}$-coordinates. When the difference between two consecutive registered points at time $t_{1}$ and $t_{2}$ exceeds $33.4 \mathrm{~ms}$, the size of the gap can be calculated by

$$
\text { gap size }=\frac{t_{2}-t_{1}-0.033}{0.033}
$$

which is then rounded to the nearest integer number. Given 2 measurements $\left(x_{1}, y_{1}\right)$ and $\left(x_{2}, y_{2}\right)$ at $t_{1}$ and $t_{2}$, the gap size missing points $\left(x^{k}, y^{k}\right)$ at time $t^{k}, k=1, \ldots$, gap size, are interpolated as follows:

$$
\begin{aligned}
& t^{k}=t_{1}+k \cdot \frac{t_{2}-t_{1}}{\text { gap size }+1} \\
& x^{k}=x_{1}+k \cdot \frac{x_{2}-x_{1}}{\text { gap size }+1} \\
& y^{k}=y_{1}+k \cdot \frac{y_{2}-y_{1}}{\text { gap size }+1}
\end{aligned}
$$

\section{Extreme Values}

The amount of data representing nonoptimal pursuing is considerable, e.g. ideal optimal horizontal pursuit would consist in y-coordinates of only $0^{\circ}$. In order to determine which y-values (x-values) can be used for analysis of horizontal (vertical) eye movements, outliers are calculated by means of the median absolute deviation, a method from robust statistics [7]. The robust mean $\bar{y}$ is given by the median of all values $y_{i}, i=1,2, \ldots, \mathrm{n}$ :

$$
\bar{y}=\operatorname{median} y_{i}
$$

and the robust standard deviation is determined by:

$$
\sigma=1.4826 \cdot \text { median }\left|y_{i}-\bar{y}\right|
$$

Then, we only retain those $y_{i}$ with $\left|y_{i}-\bar{y}\right| \leq \sigma$ for further consideration. In a similar manner, $x$-values are filtered to determine those useful for analysis of vertical eye movements.

\section{Calculation of Tracking Performance Values}

The remaining data can now be used for calculation of tracking performance values like the number, direction and velocity of saccades. The slow phases are distinguished from the fast phases (saccades) by applying a velocity threshold of $40 \%$ for saccades [8]. The velocity is determined by

$$
v=\frac{x_{2}-x_{1}}{t_{2}-t_{1}}
$$

for two consecutive measurements $x_{1}$ and $x_{2}$ at time $t_{1}$ and $t_{2}$ (after interpolation).

The same method described for outlier determination of $\mathrm{x}$ - and $\mathrm{y}$-coordinates is applied to exclude outliers in the estimated velocities.

\section{Examples of Registrations before and after Algorithm Application}

Figure 3 shows the horizontal smooth pursuit movements of 4 different children aged between 1.5 and 7 years for a stimulus with a velocity of $15 \%$. The upper curves correspond to raw data, the lower curves show the result of applying our algorithm.

\section{Discussion}

We present a simple algorithm for POG data analysis based on an extrapolation of missing values due to blinking and an exclusion of outliers using the robust mean and standard deviation. Four graphical examples of registered POG illustrate the performance of the algorithm. It can be seen that all curves get smoother after applying our algorithm. Data analysis systems for POG registration are normally integrated in the software package for POG registration. Since the algorithms used for data analysis are proprietary, data interpretation may be difficult. Our algorithm allows a quick data analysis and will help to better interpret and analyze POG data.

\section{Acknowledgments}

This work was supported by the Swiss National Science Foundation (Bern, Switzerland), grant 3200-052503.97/1, and the OPOS Foundation (St. Gallen, Switzerland).

Ophthalmologica 2003;217:320-324 


\section{References}

5 Valmaggia C, Charlier J, Gottlob I: Optokinetic nystagmus in patients with central scotomas in age related macular degeneration. $\mathrm{Br} \mathrm{J} \mathrm{Oph-}$ thalmol 2000;85:169-172.

6 Doane MG: Interaction of eyelids and tears in corneal wetting and the dynamic of the normal human eyeblink. Am J Ophthalmol 1980;89: 507-516.

7 Rousseeuw PJ, Leroy AM: Robust Regression and Outlier Detection. New York, Wiley, 1987.

8 Lengyel D, Weinacht S, Charlier J, Gottlob I: The development of visual pursuit during the first month of life. Graefe's Arch Clin Exp Ophthalmol 1998;236:440-444.
4 Prechtl HFR: The behavioural states of the newborn infant. Brain Res 1974;76:185-212.

Buquet C, Charlier JR: Evaluation of sensory visual development based on measures of oculomotor responses; in Vital-Durand F, Atkinson J, Braddick OJ, (eds): Infant Vision. New York, Oxford University Press, 1996, pp 291Med Biol Eng Comput 1994;32:197-204.
Charlier J, Buquet C, Desmidt A, Querleu D:

Application de la technique photo-oculograp que à l'étude de la poursuite visuelle au cours des premiers mois de la vie. Bull Soc Ophtalmol Fr 1993;11:973-978.

Ruetsche/Baumann/Jiang/Mojon 